\title{
On the nature of metal-carbon bonding: AIM and ELF analyses of MCHn (n=1-3) compounds containing early transition metals
}

\author{
Isaac Vidal, Santiago Melchor and José A. Dobado*
}

Grupo de Modelización y Diseño Molecular. Departamento de Química Orgánica.

Facultad de Ciencias. Universidad de Granada. Granada, E-18071, (Spain)

* Author whom correspondence should be addressed.

E-mail: dobado@ugr.es 
Figure S-1. Definition of the bending angle $\gamma$ and the orientation vector $\boldsymbol{A}$. Due to the different symmetries of methylidynes, methylidenes and methyl-metals, and for a comparison of the rocking of the $\mathrm{CH}_{\mathrm{n}}$ group, the bending angle $\gamma$ is defined for all structures as the angle formed between the M-C bond and the vector $\boldsymbol{A}$. Vector $\boldsymbol{A}$ is defined as the vector originating on the carbon atom, which subtends the same angle with all $\mathrm{C}-\mathrm{H}$ bonds, with the minimum value. For methylidynes $\mathrm{MCH}$, this vector lies along the $\mathrm{C}-\mathrm{H}$ bond, for methylidenes $\mathrm{MCH}_{2}$ (a), $\boldsymbol{A}$ coincides with the bisectrix of the $\mathrm{HCH}$ angle and, for the methyl-metals $\mathrm{MCH}_{3}$ (b), it points in the opposite direction of the pyramidalization POAV1 vector, defined by Haddon. ${ }^{1}$ Therefore, both $C_{\propto \infty}$ and $C_{s}$ geometries were tested for methylidyne compounds, the $C_{2 v}$ and $C_{s}$ ones for the methylidenes, and the $C_{3 v}$ and $C_{s}$ ones for methyl-metal derivatives, corresponding to linear $\left(\gamma=180^{\circ}\right)$ and bent $\left(\gamma<180^{\circ}\right)$ structures, respectively.
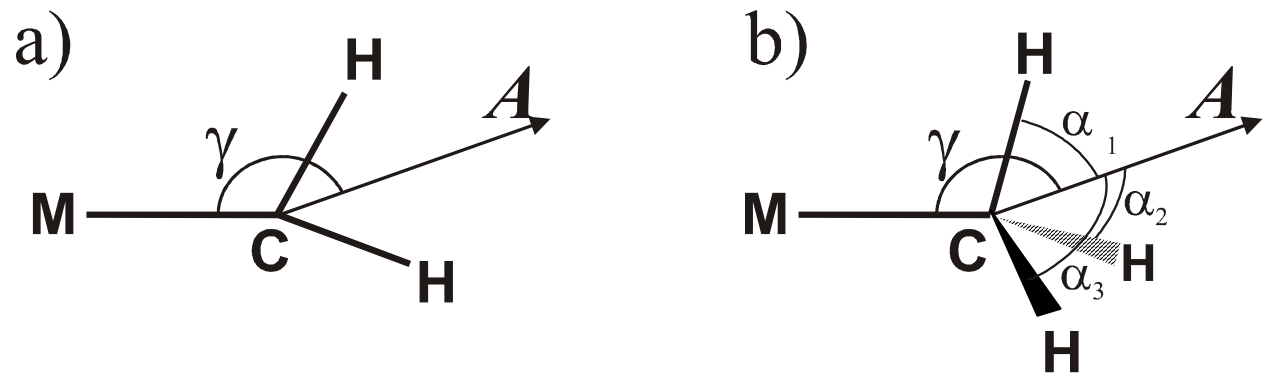

\footnotetext{
${ }^{1}$ Haddon, R.C. J. Phys. Chem. 1987, 91, 3719.
} 
Table S-2. Comparison between experimental and calculated metal-carbon bond distances $(\AA)$ for the ground state of methylidynes, methylidenes and methyl-metals where experimental data is available at different basis sets and theoretical levels.

\begin{tabular}{|c|c|c|c|c|c|}
\hline & & \multirow[t]{2}{*}{ Basis set } & \multicolumn{3}{|c|}{ Method } \\
\hline & & & B3LYP & MP2 & CISD \\
\hline \multirow[t]{3}{*}{$\mathrm{KCH}\left(C_{\infty}\right)$} & Calcd. $\left({ }^{3} \Sigma^{-}\right)$ & BS2 & 2.530 & 2.552 & 2.560 \\
\hline & & BS3 & 2.523 & 2.536 & 2.550 \\
\hline & Exptl. ${ }^{a}\left({ }^{3} \Sigma^{-}\right)$ & & 2.530 & & \\
\hline \multirow[t]{5}{*}{$\mathrm{TiCH}\left(C_{\infty V}\right)$} & Calcd. $\left({ }^{2} \Sigma^{+}\right)$ & BS1 & 1.700 & 1.699 & 1.695 \\
\hline & & BS2 & 1.688 & 1.717 & 1.698 \\
\hline & & BS3 & 1.686 & 1.703 & 1.691 \\
\hline & & BS4 & 1.686 & - & 1.691 \\
\hline & Exptl. ${ }^{b}\left({ }^{2} \Sigma^{+}\right)$ & & 1.728 & & \\
\hline \multirow[t]{5}{*}{$\operatorname{VCH}\left(C_{\infty}\right)$} & Calcd. $\left({ }^{3} \Delta\right)$ & BS1 & 1.605 & 1.748 & 1.662 \\
\hline & & BS2 & 1.682 & 1.787 & 1.662 \\
\hline & & BS3 & 1.697 & 1.752 & 1.650 \\
\hline & & BS4 & 1.697 & - & 1.647 \\
\hline & Exptl. ${ }^{c}\left({ }^{3} \Delta\right)$ & & 1.703 & & \\
\hline \multirow[t]{5}{*}{$\mathrm{CrCH}_{2}\left(C_{2 v}\right)$} & Calcd. $\left({ }^{5} \mathrm{~B}_{1}\right)$ & BS1 & 1.897 & 1.954 & 1.961 \\
\hline & & BS2 & 1.912 & 1.942 & 1.958 \\
\hline & & BS3 & 1.910 & 1.933 & 1.954 \\
\hline & & BS4 & 1.910 & - & 1.957 \\
\hline & Exptl..$^{d, e}$ & & 1.9 & & \\
\hline \multirow[t]{3}{*}{$\mathrm{KCH}_{3}\left(C_{3 v}\right)$} & Calcd. $\left({ }^{1} \mathrm{~A}_{1}\right)$ & BS2 & 2.677 & 2.677 & 2.683 \\
\hline & & BS3 & 2.671 & 2.669 & 2.676 \\
\hline & Exptl..$^{f, e}$ & & 2.633 & & \\
\hline \multirow[t]{3}{*}{$\mathrm{CaCH}_{3}\left(C_{3 v}\right)$} & Calcd. $\left({ }^{2} \mathrm{~A}_{1}\right)$ & BS2 & 2.373 & 2.410 & 2.416 \\
\hline & & BS3 & 2.357 & 2.386 & 2.393 \\
\hline & Exptl. ${ }^{g}\left({ }^{2} \mathrm{~A}_{1}\right)$ & & 2.359 & & \\
\hline
\end{tabular}

${ }^{\mathrm{a}}$ See Ref. 30. ${ }^{\mathrm{b}}$ See Ref. 33. ${ }^{\mathrm{c}}$ See Ref. $34 .{ }^{\mathrm{d}}$ See Ref. $35 .{ }^{\mathrm{e}}$ Electronic state not reported experimentally.

${ }^{\mathrm{f}}$ See Ref. 31. ${ }^{\mathrm{g}}$ See Ref. 32. 
Figure S-3. Comparative ELF isosurfaces computed at a 0.75 value for $\left[\mathrm{CH}_{n}\right]^{-}$anion and $\mathrm{CH}_{\mathrm{n}}$ neutral species (n=1-3) obtained from the B3LYP/6-311++g(3df,2p) electronic structure. Spin multiplicity is indicated in parentheses.

\section{ANION}

\section{CH}
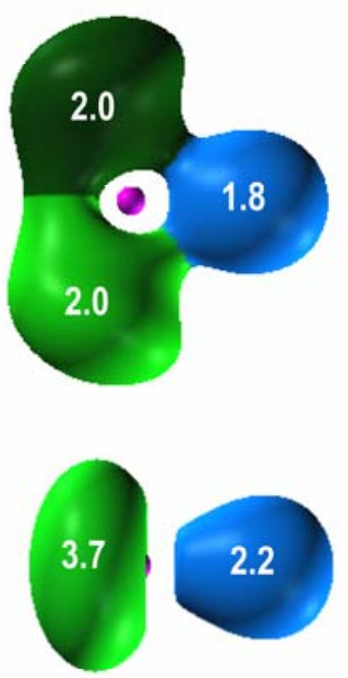

$\mathrm{CH}_{2}$
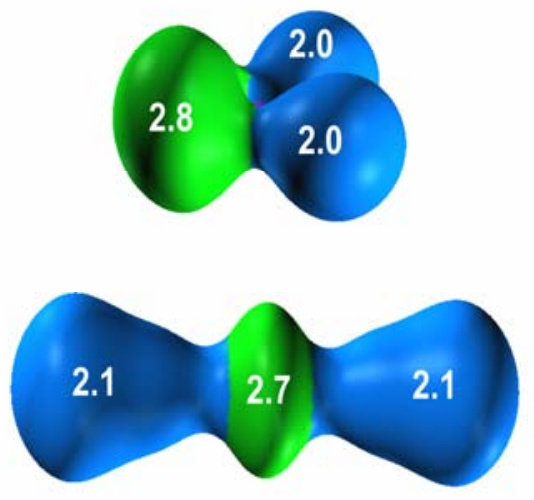

$\mathrm{CH}_{3}$

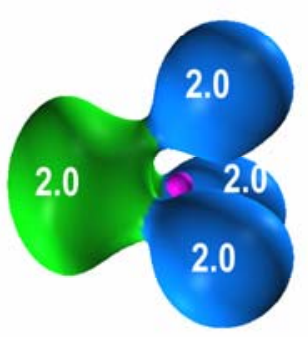

(1)

(3)

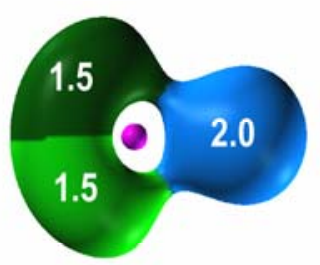

(2)

(4)

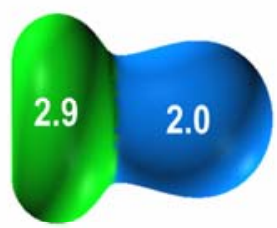

(2)

(4)

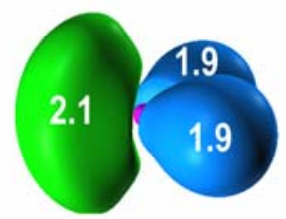

(3)

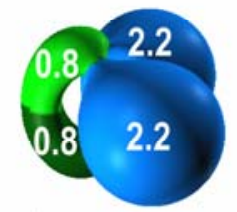

(1)

(2)

(3)

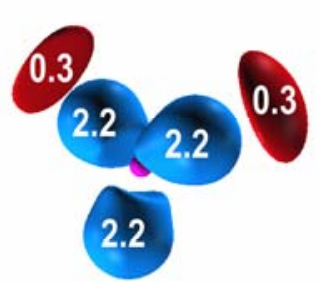

0.3

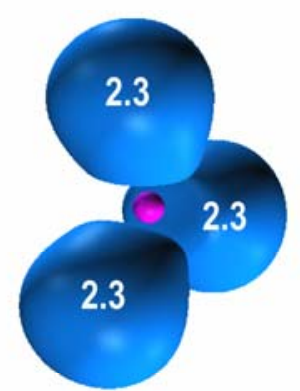

(4) 
Figure S-4. Laplacian $\nabla^{2} \rho(r)$ contour maps, for compounds 1-7(a-c) computed at the B3LYP/6-311++G(3df,2p) level. The contours values correspond to $\pm 0.02, \pm 0.04, \pm 0.08$, $\pm 0.2, \pm 0.4, \pm 0.8, \pm 2.0, \pm 4.0$ and $\pm 8.0 \mathrm{e} \cdot \mathrm{a}_{0}{ }^{-5}$, where the dashed lines indicate negative values.

\section{$\mathrm{KCH}$}

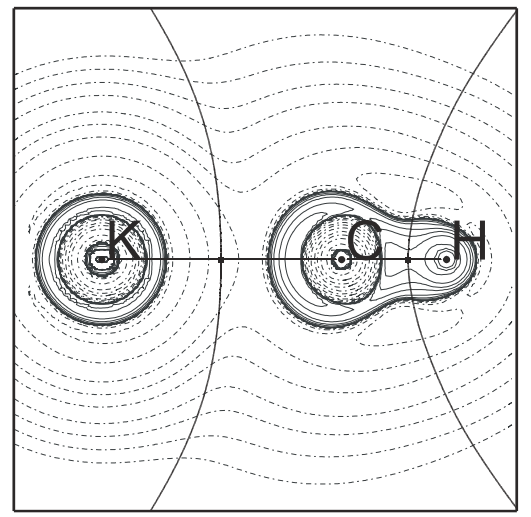

\section{$\mathrm{ScCH}$}

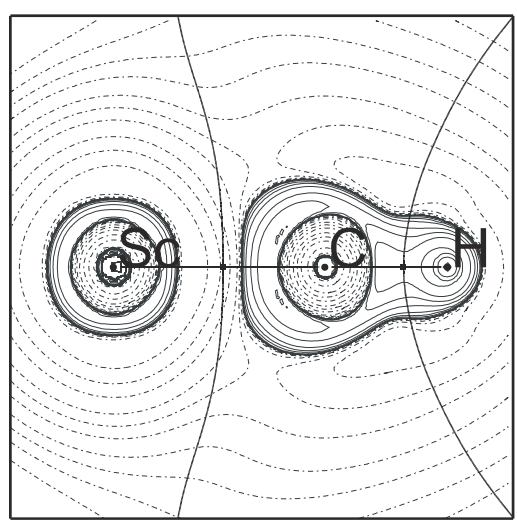

$\mathrm{CrCH}$

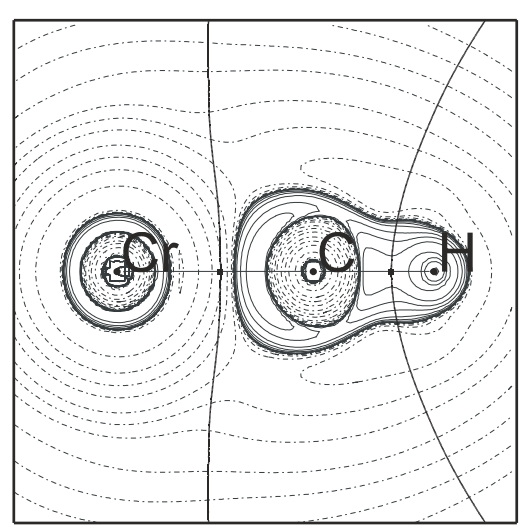

$\mathrm{CaCH}$

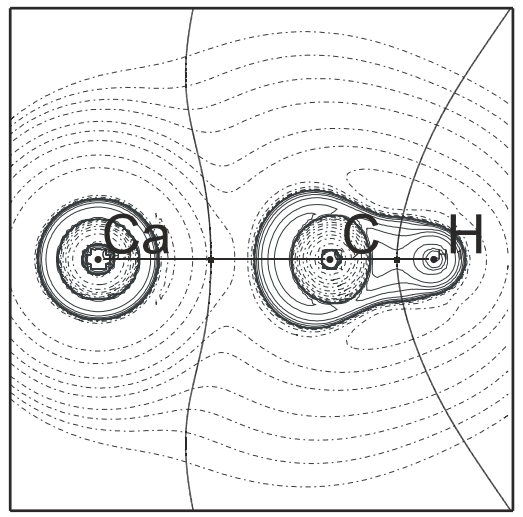

$\mathrm{VCH}$

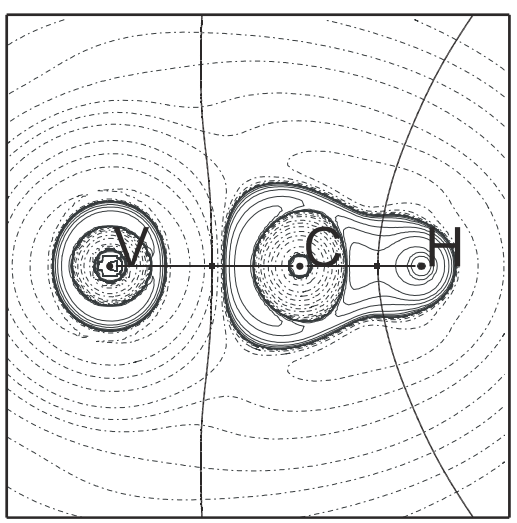

$\mathrm{TiCH}$

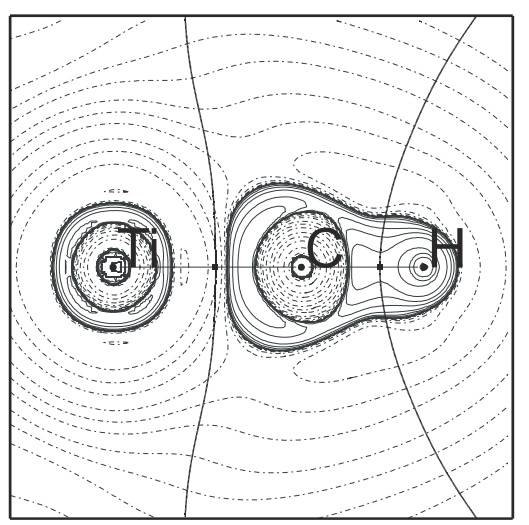

\section{$\mathrm{MnCH}$}

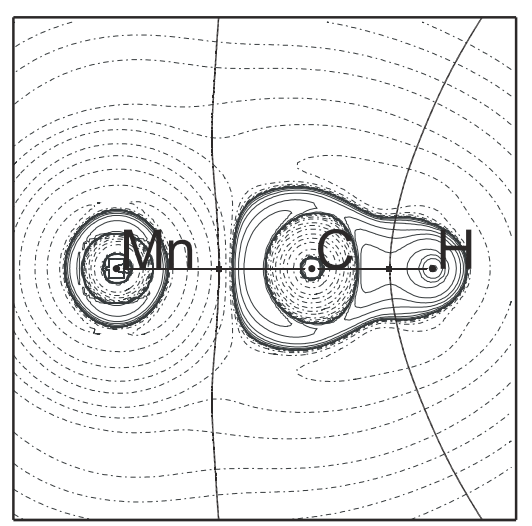


Figure S-4: (continued)

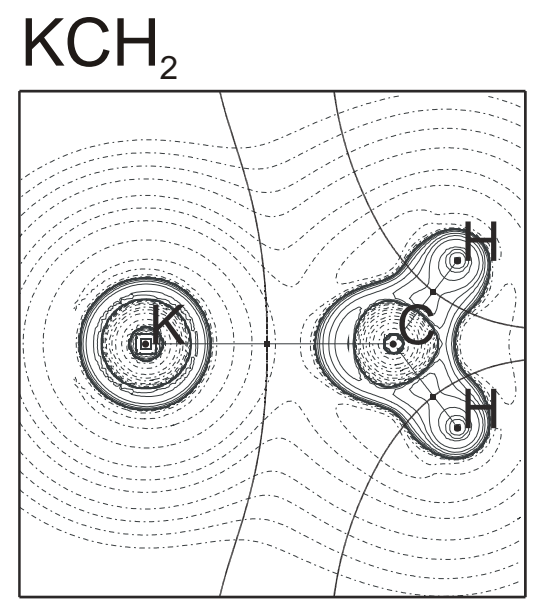

$\mathrm{CaCH}_{2}$
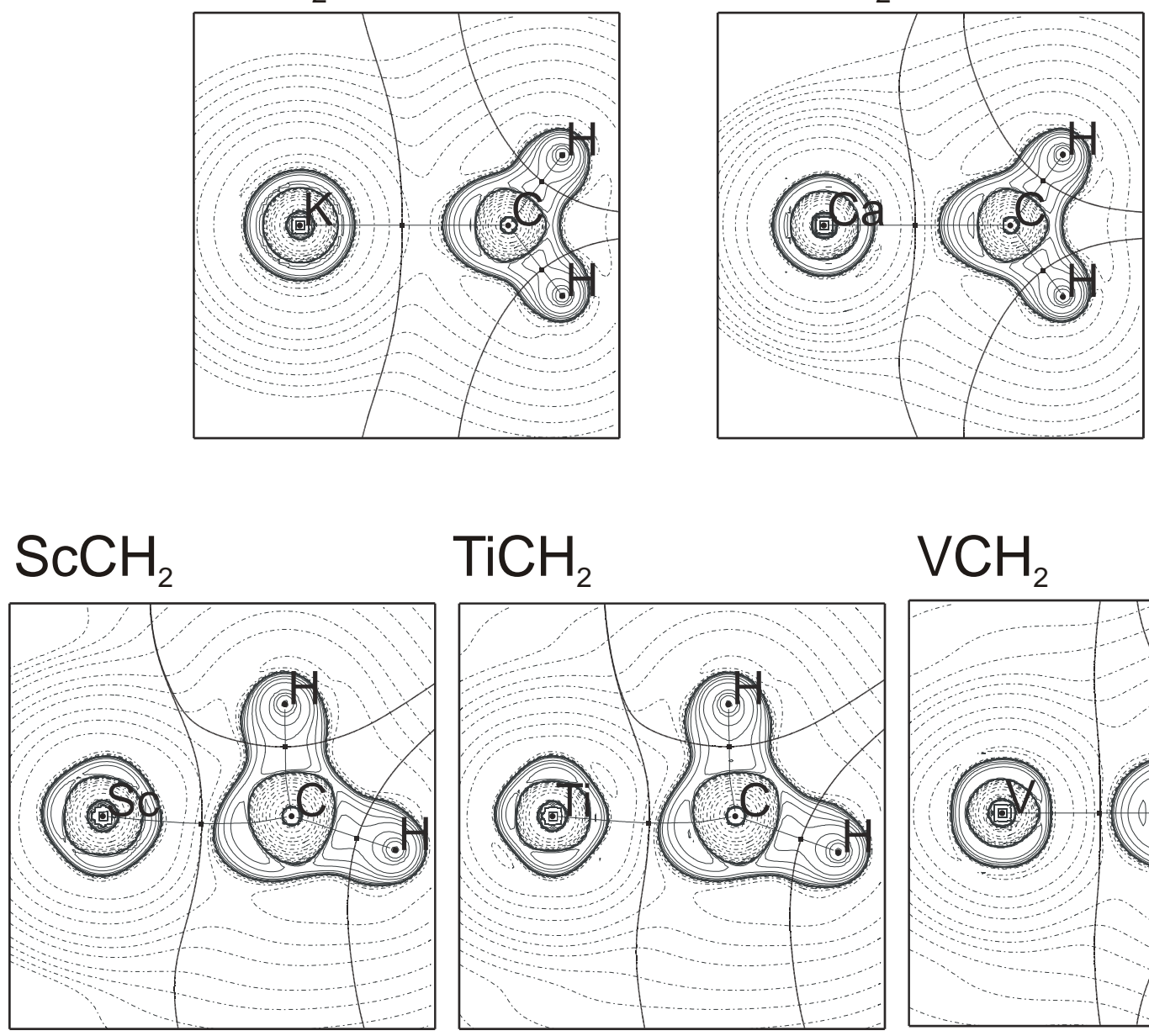

$\mathrm{TiCH}_{2}$

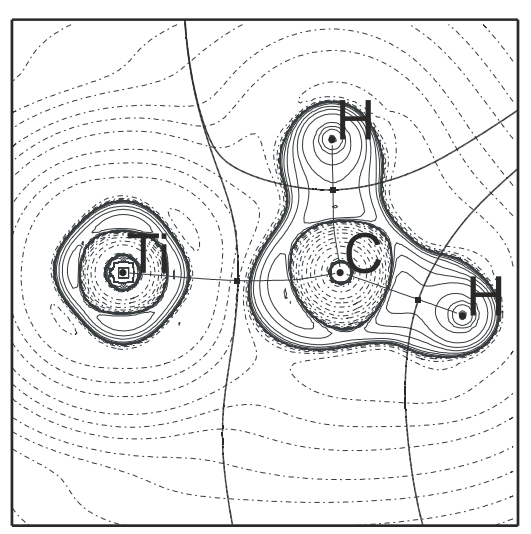

$\mathrm{VCH}_{2}$

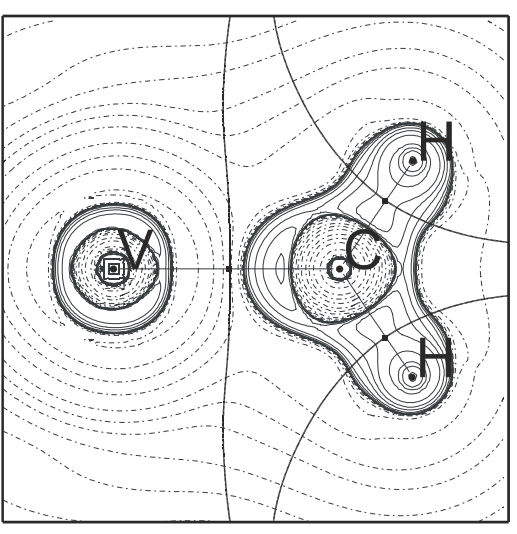

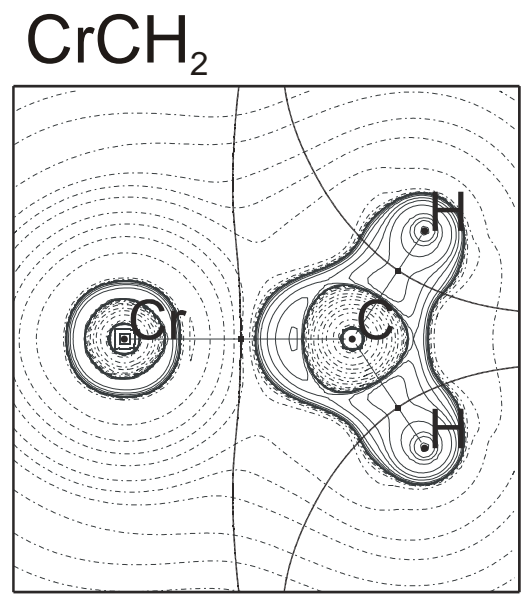

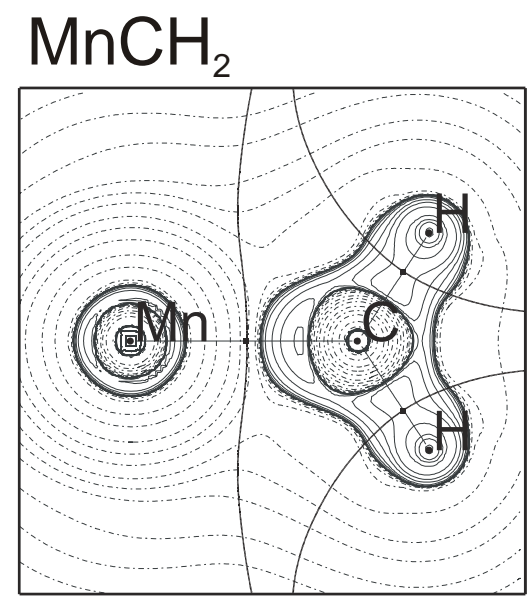


Figure S-4: (continued)

$\mathrm{KCH}_{3}$

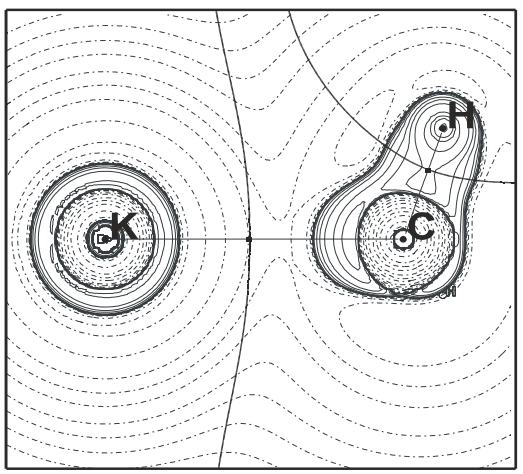

$\mathrm{ScCH}_{3}$

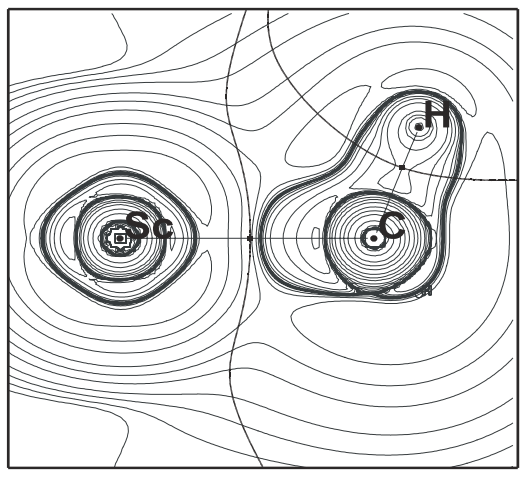

$\mathrm{CrCH}_{3}$

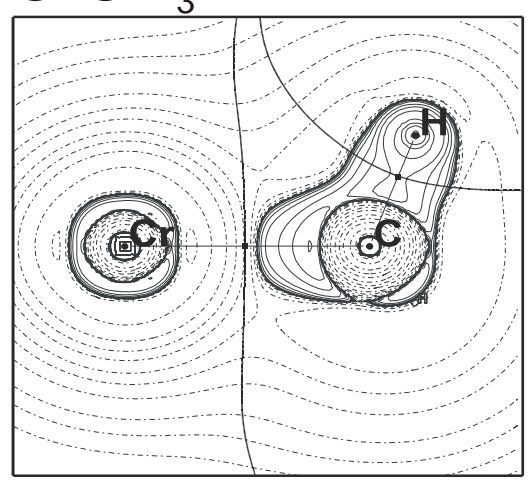

$\mathrm{CaCH}_{3}$

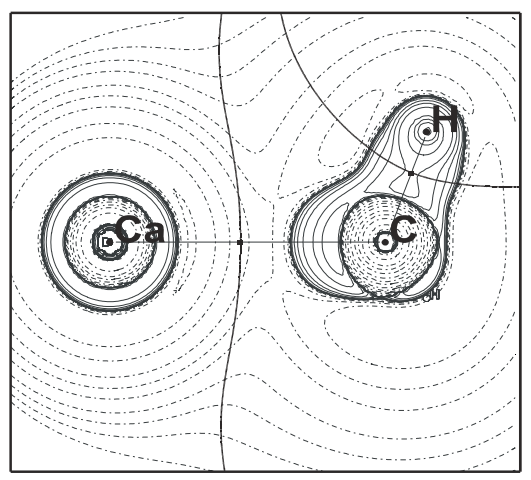

$\mathrm{VCH}_{3}$

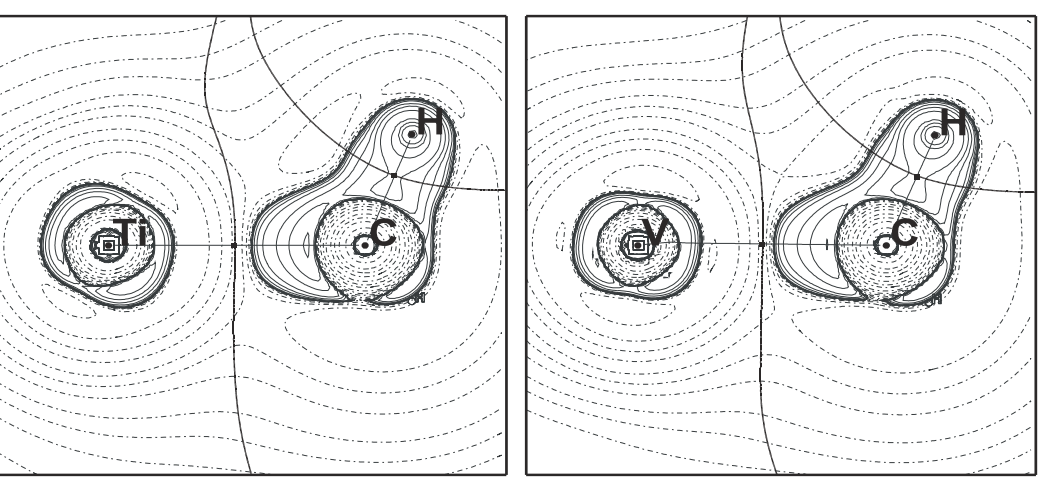

$\mathrm{MnCH}_{3}$

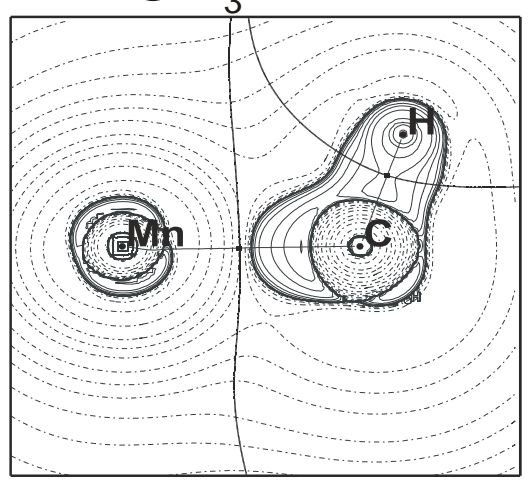


Figure S-5. ELF isosurfaces and pairing basin populations for the $\mathrm{MCH}_{3}$ overall minima. 1c-7c, computed at a value of 0.75 . Asterisks denote the existence and the population of low-pairing basins with values below 0.75 . The colour convention represents core basins in magenta, and the remaining valence basins are classified depending on the number of connections to core nuclear basins (synaptic order): red for monosynaptic, green for disynaptic and cyan for disynaptic hydrogenated basins.
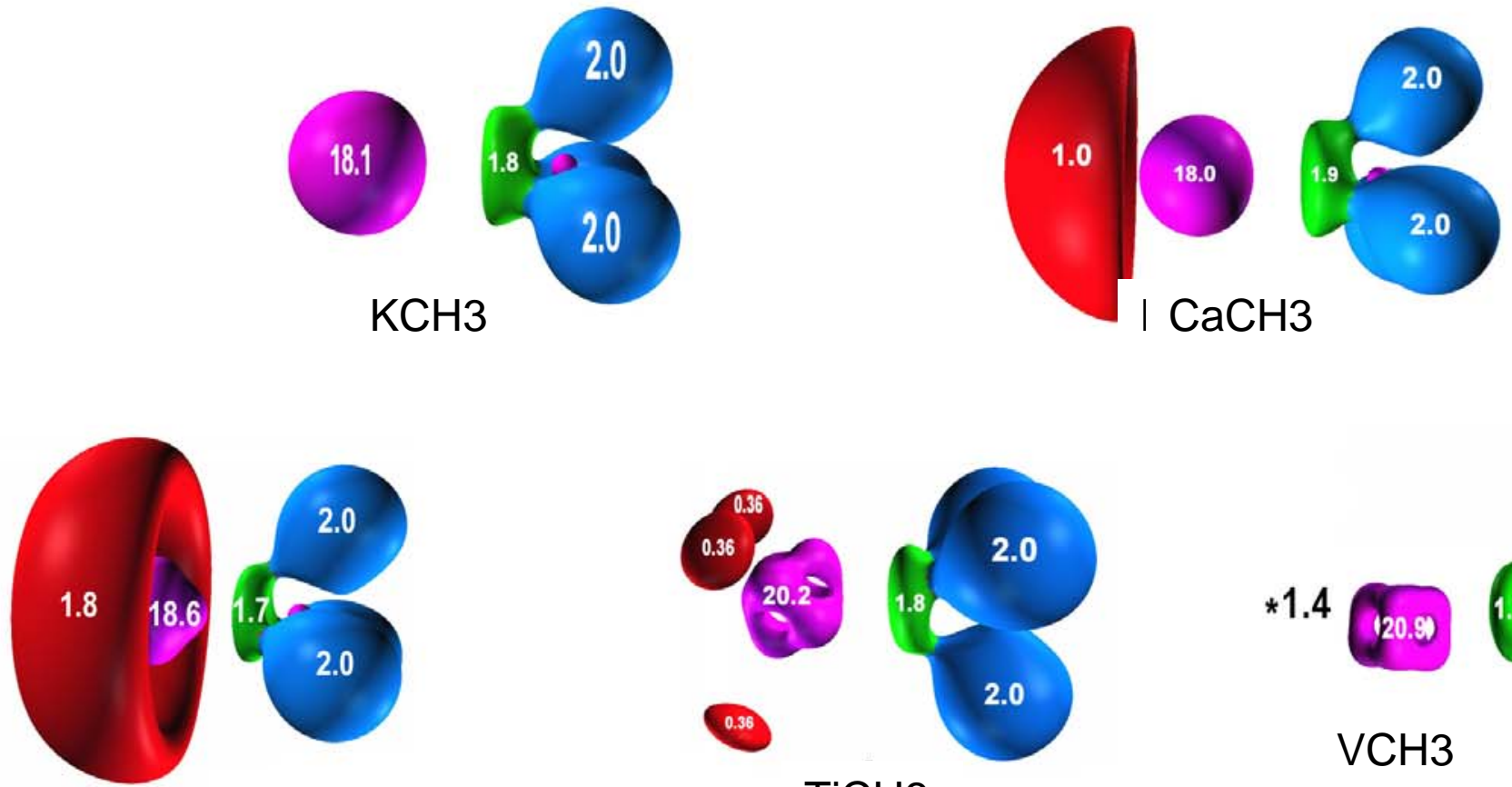

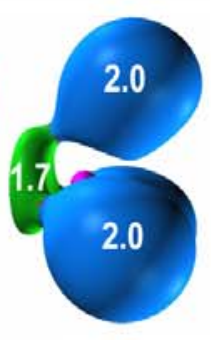

$\mathrm{ScCH} 3$

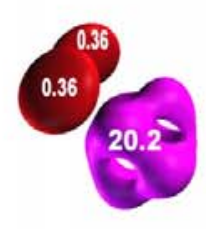

0.36

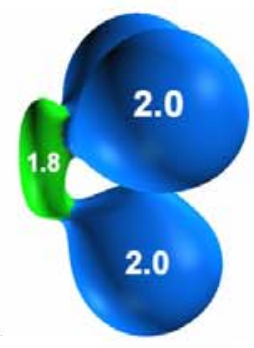

$\mathrm{TiCH} 3$

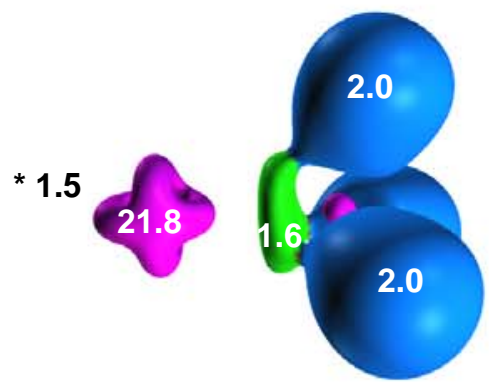

$\mathrm{CrCH} 3$

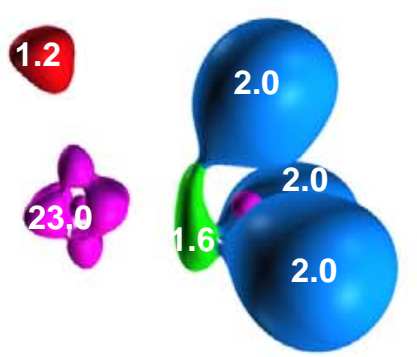

$\mathrm{MnCH} 3$ 